\title{
A review on the cognitive functions of basal ganglia, amygdala, hippocampus, habenula, nucleus accumbens, cerebellum, and pineal gland
}

\author{
Paolo Lissoni*, Giusy Messina, Giorgio Porro, Carlo Gavazzeni, Roberto Trampetti, Olga Tartarelli, Rosanna Tartarelli, Alberto Caddeo \\ and Giuseppe Di Fede \\ Institute of Biological Medicine, Milan, Italy
}

\begin{abstract}
Until few years ago several brain areas, including the basal ganglia and cerebellum, were belived to play a role only in the control of motor activity, whereas most recent researches have shown that they are also involved in cognitive functions, and in the connection between unconscious and conscious emotional life. The main brain structure responsible for the response to the different environmental and affective stimulations is represented by the amygdala, namely by its basolateral nucleus, through its interactions with the hippocampus, the main site of long term memory. An excessive sensibility of amygdala would tend to respond on the basis of previous similar previous experiencs, with a following decline in the freedom of subjects, since the freedom requires a possibility of choise irrespectively of the past experiences. The sensitivity of amygdala is namely under a neurohypophyseal control, since it is abnormally enhanced by vasopressin, whereas it is reduced by oxytocin. Moreover, brain opioid system and catecholamines enhance the amygdala sensitivity, whereas it is reduced by brain cannabinergic system and the pineal gland, which is the only organ of human body able to modulate the biological systems according to the different universal conditions. The different sensitity of amygdala would allow different nervous connections. In more detail, an excessive response of amygadala to the various stimulations would namely activate the nucleus accumbens at mesolimbic site, and the putamen at basal ganglion level, whereas a less sensitivity of amygdala would activate the habenula, which is strickly connected to the pineal function at epithalamic site and caudatum, by inducing a behavioral response more determined by a cognitive conscious decision. Moreover, the functional axis constituted of amygdala-cannabinergic system-oxytocin-pineal gland-habenula is involved in self-conscious processes, love and pleasure perception, whereas the amygdala-opioid system-vasopressin-nucleus accumbens is active in stress, anxiety, and depressive conditions. Then, the neurobiochemistry reponsible for the status of freedom would be the same of mind expansion, pleasure and love. The end-result of the overall nervous interactions may be identified into the activation of mirrorneuron system, which plays a fundamental role in learning and self-consciousness generation. Mirror neuron activity is stimulated by cannabinoids, oxytocin and the pineal hormone melatonin, whereas it is inhibited by mu-opioid agonists, vasopressin, and catecholamines. Then, because of the essential role of mirror-neurons in self-consciousness processes, it is possible to conclude that the expansion of consciousness is associated with the feeeling of love and pleasure perception, whereas it is opposited by the overall conditions characterized by a decline in the status of consciousness, including stress, anxiety and depression.
\end{abstract}

\section{Introduction}

Until few years ago, several brain structures, such as the basal ganglia and cerebellum, were belived to play a role only in the control of motor activity. On the contrary, more recent experimental studies have shown that basal ganglia system and cerebellum itself are also involved in cognitive functions by integrating between them conscious and unconscious stimulations and informations. The main brain regions involved by the control and cognitive and emotional life are consisting of the basal ganglia, the amygdala through its connnection with the hippocampus, the habenula, the nucleus accumbens, the ventral tegmental area (VTA), the locus coeruleus, the nuclei of raphe, brain pons and cerebellum itself [1-6]. Moreover, the emotional and cognitive life is depending on the main neurotransmission pathways. The VTA is the main source of the dopaminergic transmission, but it is consisted also of glutaminergic and gabaergic neurons. VTA is also under a stimulatory control mediated by the glutaminergic pathway. The nucleus accumbens is also a dopaminergic system. VTA-induced release of dopamine namely activates the nucleus accumbens. Then, the mesolimbic dopamine system may be considered as constituted of VTA and nucleus accumbens. The nucleus accumbens may be also activated by both ventral subiculum nucleus of the hyppocampus, and basolateral amygdala, and it may activate both the noradrenergic pathway arising from the locus coeruleus, and mesolimbic dopamine system [2]. The mesolimbic system is namely involved in brain reward circuit by promoting reward-seeking behavior, including drug-related reward. The locus coeruleus is the origin of the noradrenergic pathway, which is involved in stress conditions and aggressive behavior. Finally, the nuclei of raphe are the main origin of the serotoninergic system. The basal ganglia are a set of interconnected sub-cortical structure, consisting of the caudate nucleus, also called striatum, lenticular nucleus, the substantia nigra, the sub-thalamic nucleus, and the peduncolopontine nucleus [1]. The lenticular nucleus is sub-divided into an external pars, the putamen, and a medial pars, the globu spallidus (or pallidum). The basal ganglia represent the area of brain, through which the informations coming from various cortical regions are transported to the motor cortex by the ventrolateral thalamus.

${ }^{\star}$ Correspondence to: Paolo Lissoni, Institute of Biological Medicine, Milan, Italy, E-mail: paolo.lissoni@gmx.com

Key words: amygdala, cannabinoid system, cognitive functions, habenula, neurohypophysis, nucleusaccumbens, opioid system, oxytocin, pinealgland

Received: April 01, 2020; Accepted: April 13, 2020; Published: April 17, 2020 
The role of the basal ganglia in cognition, working memory, and learning is still to be better defined. In any case,it is now known that they are involved not only in motor control, but also in emotions, mood, learning, and obsessive-compulsive disorders, with different activity in relation to each single neuronal nucleus. Recently, it has also been demonstrated the fundamental role of the epithalamus in the modulation of the psychospiritual life. The epithalamus substantially consistes of the habenula, and the pinelgland. The stria medullaris and the lateral hypothalamus may be also included within the epithalamus brain region. The habenula is sub-divided into medial and lateral habenula, which are provided by both different and complementary different activities [3]. Medial habenula receives its main imput from the limbic system, and sends outputs to the interpeduncolar nucleus, which projects to the serotoninergic system of raphe. On the other side, the lateral habenula receives its major input from the basal ganglia, and projects to both serotoninergic system of raphe and the dopaminergic one of the VTA. At present, no clear direct connection between dopaminergic and serotoninergic systems have been documented. The habenula would represent the main connection among limbic system, basal ganglia, and nucleus accumbens.Then, in other words, the habenula would constitute an integration among limbic system, basal ganglia, and nucleus accumbus, which play an important role in modulating the connections between limbic system and basal ganglia. Finally, both habenula and nucleus accumbens are connected with the amygdala-hippocampal system, and amygdala would represent the main nervous structure primarily involved in the response to the different social and environmental stimulations, by integrating consciuos and unconscious aspects of human behaviors [6].

\section{The cognitive function of the basal ganglia}

Each single nucleus of the basal ganglia seems to exert different cognitive and emotions activities, even though no schematic classification may be definely proposed. One of the main investigated nuclei is the caudate nucleus $[4,5]$. Despite the controversial results, it is possible to conclude that the caudate nucleus may contribute to behavior through the excitation of correct action schemas and the selection of the more appropriate manner to respond based on an evaluation of action-outcomes. From this point of view, the function of the caudate nucleus is different from that played by putamen, which in a less evoluted manner has appeared to exert cognitive functions more limited to stimulus-response, then to the pavlovian reactions [4]. Then, within the cognitive processes, putamen would be involved in the only regulation of the stimulus-response relation, as well in obsessivecompuilsive disturbances, since suggested by the evidence of obsessivecompulsive disorders in patients with lesions of putamen [7]. On the contrary, the main role of the caudate nucleus would consist of social informations into coding of social actions and reward. The ventral pallidum contains a gabaergic system, which may inhibit mesolimbic dopamine system, with a possibile inhibitory control on pleasure perception and reward circuit [8]. Finally, within the basal ganglion structure, the substantia nigra is a mid brain dopaminergic system, and it would constitute the primary imput into the basal ganglion circuit [9]. Another area near to the basal ganglia is the claustrum, an irregular neuronal structure between neocortex and putamen. It has appeared to play a role in the integration of the various conscious perceptions and cognitions [10].

\section{Habenula as the integrator between limbic and basal ganglion systems}

Habenula has recently been rediscovered as a fundamental brain area involved in the modulation of emotions and consciousness states $[3,11,12]$. Medial and lateral habenula may be distinguished anatomically, but not functionally, even though they exert some preferential activities. In any case, most studies have been performed in an attempt to analyze the functionless of the lateral habenula, which has been proven to be involved in negative reward when we do not receive an expected reward, as well as in pleasure perception, selfmotivation, memory processes and their translation into action, fear, aggressive behavior, reinforcement, and addiction. Medial habenula has appeared to be also involved in the emotional control, including mood, anxiety, response to stress, working memory, drug addiction, and nicotine dependency. By synthetizing, the habenula modulates multiple brain systems, by representing the only integrator system between limbic system and basal ganglia.

\section{The role of amygdala in the psychospiritual life}

Amygdala plays a fundamental role in the integration of the various area of the limbic system, since its sensitivity influences the type of response to the different emotional stimulations arising from several cognitive and neurovegetative areas, then from both conscious and unconscious informations, by comparing the new stimulations with respect to the memory of previous similar experiences through its connections with the hippocampus, whose fundamental role in long-term memory storage is well known, namely with the ventral subiculum of hippocampus [13]. The amygdala response is related to the activation of its basolateral nucleus. Then, because of the fundamental role of the sensitivity of amygdala basolateral nucleus in influencing the whole brain response, from an imagination point of view the basolateral nucleus of amygdala could be defined as the clitoris of brain, since clitoris is the most innervated and sensitive anatomic structure of the human body. Amygdala is also connected to the hypothalamus, and the medial dorsal nucleus of thalamus. The emotional stimulations influence the activity of amygdala by acting on the basolateral nucleus, which may activate the central nucleus of amygdala, which projects to the mesolimbic region. The sensitivity of amygdala is under a complex nervous, neuroendocrine, and endocrine regulation. An excessive response of amygdala to the various emotional stimulations would allow to constantly react on the basis of the memory of similar emotional experiences in the past, by making the human subject as unable to be free to live new experiencesin a new manner. Then, freedom itself requires a well defined neurobiochemical mediation.

\section{The cognitive functions of cerebellum and pons}

Even though very few evidences are available up to now, both pons and cerebellum have been proven to influence the cognitive processes $[14,15]$. Cerebellum seems to exert cognitive functions through its connections with both cortex and basal ganglia, and in memory processes, namely those involving the neurovegetative unconscious states in relation to the various organs of human body. Brain pons would be also involved in several sensorial and cognitive functions, by realizing an integration between cerebrum and cerebellum [15].

\section{The nervous circuits of amygdala-habenula-pineal and amygdala-putamen-accumbens}

Both unconscious and conscious behaviors are the results of multiple brain interactions, but on the same time it is true that some neuronal circuits are more linked to the conscious life, and others to the unconscious one. From this point of view, according to the various experimental and often contradictory results, it is possible to identify two major neuronal circuits, the amygdala-habenula-pineal (AHAP) 
functional axis, and the amygdala-nucleus accumbens-putamen(AAP) functional circuit $[2,3]$. Then, amygdala would play a fundamental role in distinguish two major essential human psychospiritual profiles, by depending on its response to the various stimulations, as well as on its prefential activation of the habenula, or the nucleus accumbens $[4,16]$. AHAP axis would be more connected to the conscious life, then to value-based decisions and to the freedom in the choices of our life, whereas the AAP neuronal circuit is more linked to the unconscious world of the human mind. AHAP axis seems to be more connected to the serotoninergic system, whereas the AAP one to the noradrenergic system, and both neuroendocrine circuits are involved in the modulation of the dopaminergic pathway and its interactions. The fundamental role of the habenula is also justified by its relation with the pineal gland, the only human organ able to transduce the environmental electromagnetic conditions into a modulation of the psychobiological response [17]. The amygdala response to the various stimulations would substantially depend on its preferential activation of the habenula, or the nucleus accumbens. Habenula would mediate the main connection between amygdala and limbic system, whereas the nucleus accumbens could mediate the connections between amygdala and basal ganglia. Several types of alterations involving amygdala, basal ganglia, nucleus accumbens, and habenula have been described in psychiatric disorders, including anxiety, schizofrenia, depression, and obsessive-compulsive disturbances. The relationships between amygdala and the pineal-habenular complex would be important in determing an adequate emotional response to the different environmental and social conditions. In fact, the pineal hormone melatonin (MLT), the most investigated pineal hormone, has appeared to be a potential regulator to memory formation and other cognitive processes through its influence amygdala sensitivity, hippocampusamygdala interactions, and habenula activity $[17,18]$. Moreover, it has to be taken into consideration that the sensitivity of amygdala is also under a complex neuroendocrine regulation [19]. In more detail, amygdala may be excessively stimulated by vasopressin (antidiuretic hormone: $\mathrm{ADH}$ ), catecholamines, $\mathrm{CRH}$, and mu-opioid agonists,, such as betaendorphin itself, whereas it is reduced by oxytocin (OT), cannabinoid agonists and MLT itself [18-20]. Moreover, both cannabinoids and MLT have appeared to stimulate the synaptic plasticity $[18,21]$. The end-result of the great variety of cognitive processes would be that on the mirro-neuron system, whose fundamental role in learning, social and affective relationships, and self-consciousness processes had been well demonstrated [21]. Both OT and cannabinoids may stimulate the mirror neuron activities, whereas $\mathrm{ADH}$, as well as catecholamines and mu-opioid agonists, may inhibit mirror neuron functions [18,22,23]. Moreover, OT inhibit the memory formation, whereas ADH stimulate the memory processes, namely the long-term ones. Then, because of the opposite effects of the two neurohypohyseal hormones $\mathrm{ADH}$ and OT on emotions, cognition, memory and behavior, it is possible to affirm that the neurohypophysis plays an important role not only in the control of water metabolism, but also in the regulation of emotions and cognitive mechanisms, because of their connections with both opioid and cannabinoid systems, the two major inter neuronal neuromodulatory circuits. ADH is more linked to the activation of the opioid system, whereas OT is connected to the cannabinoid system and to the pineal gland, because of the positive feedback mechanism occurring between OT and MLT secretions [24,25]. Finally, the pineal secretion of MLT is under a stimulatory control played by the cannabinoid agents [26].

\section{The role of the neurohypophysis in regulating brain- heart emotional connections}

It is known that the heart is under a hemodynamic and a neuroemotional regulation. The connection between hemodynamic and psychoemotional status would be mainly realized by the neurohypophysis, since its hormones may either influence brain cognitive and emotional functions, or be connected with the cardiac endocrine activity, which namely consistes of a preferential release of atrial natriuretic peptide (ANP) or endothelin-1 (ET-1) [27,28]. ANP is connected to OT by a positive feedback mechanism, since the are linked by a reciprocal stimulatory action [29]. On the same way, ADH is related to ET-1 secretion by a reciprocal stimulatory activity [30]. Then, by considering the influence of the neurohypophyseal hormones, namely on the sensitity of amygdala, as well as between pineal activity and OT secretion, we may identify two major fundamental heart-brain functional circuits, the functional brain-pineal-neurohypophys-heart axis, consisting of cannabinoid system-habenula-pineal-amygdalaOT-ANP secretions, and the functional brain-adenohypophysisneurohypophysis-heart axis, consisting of opioid system-nucleus accumbens-amygdala-pituitary-ADH-ET-1 axis [19,25]. These two fundamental biological ways are provided by both opposite and complementary functions.

\section{The pineal gland as the symbolic site of the integration between spirit and material body}

MLT is the main pineal hormone, but it could simply represent the most investigated one, since most studies concerning the pineal function and physiology are substantially limited to the only MLT secretion and activity. In more detail, MLT is only one of the four indole hormones produced by the pineal gland, consisting of MLT itself, 5-methoxytriptamine (5-MTT), the 5-methoxytryptophol (5-MTP), and the 5-metoxy-indole-acetic acid (5-MIA). The pineal indoles are released according to the light/dark circardian rhythm. The secretion of MLT is not under a hematic regulation, but under a nervous control, depending on noradrenaline release from the postgangliar fiber arising from the superior cervical ganglion, which acts on a betaadrenergic receptor expressed by pinealocytes [17]. The light inhibits noradrenaline release from the postgangliar fiber, which in contrast is stimulated by the dark, with a following generation of a light/dark circadian rhythm in MLT secretion, with higher levels during the night and lowest concentrations during the light period of the day. The circadian secretion of the other pineal indoles has been less analyzed. However, it seems that 5-MTP is mainly produced during the period of maximum light, 5-MTT in the early afternoon, and 5-MIA during the morning. Moreover, the pineal gland is the main organ responsible for the production of beta-carbolines, a group of molecules provided by antitumor, antidepressant and psychedelic properties, which would play a fundamental role in the regulation of consciousness states. In addition to the stimulatory effect of beta-adrenergic agonists, MLT secretion is also stimulated by alpha-1 agonists, alpha-2 antagonists, cannabinoid agonists, oxytocin, ANP, and VIP, whereas it is inhibited by beta-blockers, alpha- 2 agonists, and high-dose steroid, while the opioid agents may exert both inhibitory and stimulatory effects, depending on the dosage and the type of opioid agonist. The pineal gland has been proven to exert a fundamental role in the maintenance of the natural anticancer biological resistance, namely through MLT itself, 5-MTT, and beta-carbolines, and in the modulation of bodymind interactions, then the expression of self-consciousness within the biochemistry of the biological body [17]. The fundamental role of the 
pineal in regulating the status of consciousness and the expansion of mind is also confirmed by the evidence of an altered neuroendocrine pineal function in cognitive disorders, including schizofrenia, autism, and dementhia.

\section{Conclusions}

The main brain area responsible for the regulation of emotions is the limbic system, which constitutes a complex network for modulating the emotional life through its interaction with the mesolimbic system [31]. The limbic system is constituted of five major areas, consisting of limbic cortex ( cingulate gyrus and parahippocampal gyrus), amygdal, hippocampus, septal area, and hypothalamus. The mesolimbic system includes the ventral segmental area, which is the main origin of the dopamie system, and the nucleus accumbens. The integration among the various areas of the limbic system is mainly realized and determined by amygdala, whose activity is collected to the hippocampus-related memory processes, depending on its preferential connection with the nucleus accumbens, or with the habenula-pineal system $[6,32]$. Amygdala is also connected with the hypothalamus an the medial dorsal nucleus of the thalamus [33]. Epithalamus is constituted of habenula and pineal gland. Finally, the diencephalon, the area interposed between brain stem and cerebral cortex, is constituted of epithalamus, ventral thalamus with the sub-thalamic nucleus, and dorsal thalamus, often referred as the classical thalamus, with the hypothalamus as related to both limbic system and diencephalon. The thalamus represents one of the main brain areas responbile for transferring both sensorial and motor informations from the subcortical structures to the cerebral cortex, by constituting the gateway to the cortex. Then, the thalamus realizes a functional integration among cerebral cortex, cerebellum, and brain stem activities. Amygdala is also under a neurohypophyseal control, since it is excessively stimulated by $\mathrm{ADH}$, and inhibited by OT [19]. One of the main end-results of the two different brain-pituitarypineal-heart functional circuits is that concerning their influence on mirror-neuron system, whose fundamental role in self-consciousness processes, interpersonal relationships, affective behavior, social identity, and perception of the world has been well established [34]. The mirror-neuron system is activated and stimulated by the cannabinoid system-amygdala-pineal-habenula-OT-ANP functional axis, which is active in pleasure and spiritual sensitivity conditions. On the contrary, mirror-neuron system is inhibited by the stress-related opioid systemamygdala-pituitary-locus coeruleus-nucleus accumbens-ADHET-1 functional axis. The former brain-endocrine-heart functional axis has also appeared to enhance the natural biological anticancer resistance, because of the antitumor activity of the overall involved molecules, including cannabinoid agonists, pineal hormone MLT, OT and ANP, whereas the latter functional axis may predispose to cancer development, because of the protumoral activity of both $\mathrm{ADH}$ and ET-1, and the inhibitory effects of the mu-opioid agonists on the anticancer immunity [21,28,35-39]. From a phylosophic point of view, this evidence would demonstrate that the emotional behavior is not only a morality question, because of its influence on the biological response and on the anticancer resistance, which is reduced by stress, depression, pain, and aggressivity conditions, whereas it is promoted and amplified by pleasure, love, and spiritual expansion of mind $[16,18]$. Faith itself may be amplified by both MLT and OT $[18,40]$.

\section{References}

1. Albin RC, Yong AB, Penney JB (1989) The functional anatomy of basal ganglic disorders. Trends Neurosci 12: 366-375. [Crossref]

2. Carlezon WA Jr, Thomas MJ (2009) Biological substrates of reward and avversion: a nucleus accumbens activity hypothesis. Neuropharmacology 56: 122-132. [Crossref]
3. Hicosaka O, Sesack SR, Lecourtier L, Shepard PP (2008) Habenula: cross road between the basal ganglia and the limbic system. J Neurosci 28: 11825-11829. [Crossref]

4. Grahn JA, Parkinson JA, Owen AM (2008) The cognitive functions of the caudate nucleus. ProgNeurobiol 86: 141-155. [Crossref]

5. Baez-Mendoza R, Schults W (2013) The role of the striatum in social behavior. Front Neurosci 7: 233-239. [Crossref]

6. Janak PH, TyeKM (2015) From circuits to behaviours in the amygdala. Nature 517: 284-292. [Crossref]

7. Sefcsik T, Nemeth D, Janacsek K, Hoffman I, Scialabba J, et al. (2009) The role of putamen in cognitive functions: a case study. Learning Percept 2: 215-227.

8. Walsh ND, Phillips ML (2004) Interactingoutcomeretrieval, anticipation, and feedback processes in the human brain. Cereb Cortex 20: 279-281. [Crossref]

9. Zecca L, Tampllini D, Gerlach M, Rieder P, Fariello RG, et al. (2001) Substantia nigra neuromelanin: structure, synthesis, and molecular biology. MolPathol 54: 414-418. [Crossref]

10. Crick FC, Koch C (2005) What is the function of the claustrum? Philos Trans R Soc London B Bibl Sci 360: 1271-1279. [Crossref]

11. Hikosaka $\mathrm{O}$ (2010) The habenula: from stress evasion to value-based decision-making. NatRevNeurosci 11: 503-513. [Crossref]

12. Viswanath H, Carter AO, Baldwin PR, Molfese DL, Salas R (2013) The medial habenula: still neglected. Front HumNeurosci 7: 931-939. [Crossref]

13. Richter-Levin G, Akirav I (2009) Amygdala-hippocampus interaction in relation to memory. MolNeurobiol 22: 11-20. [Crossref]

14. Sullivan EV (2010) Cognitive functions of the cerebellum. Neurophysiol Res 20: 227229. [Crossref]

15. Buckner RL (2013) The cerebellum and cognitive functions: 25 years of insight from anatomy and neuro imaging. Neuron 30: 807-815. [Crossref]

16. Mohandas E (2008) Neurobiology of spirituality. Mens Sana Monogr 6: 63-80. [Crossref]

17. Brzezinsky A (1997) Melatonin in humans. N Engl J Med 336: 186-195. [Crossref]

18. Bob P, Fedor-Freybergh F (2008) Melatonin, consciousness, and traumatic stress. $J$ Pineal Res 44: 341-47. [Crossref]

19. OKhajehei M, Behroozpour E (2018) Endorphins, oxytocin, sexuality and romantic relationships. An understudied area. World J ObstetGynecol 7: 17-23.

20. Ganon-Elazar E, AkiravI (2009) Cannabinoid receptor activation in the basolateral amygdala blocks the effects of stress on the conditioning an extinction of inhibitory avoidance. $J$ Neurosci 29: 11078-11088. [Crossref]

21. Grotenhermen F (2004) Pharmacology of cannabinoids. NeuroendocrinolLett 25: 14 23. [Crossref]

22. Bachner-Melman R, Ebstein RP (2014) The role of oxytocin and vasopressin in emotional and social behaviors. HandbClinNeurosci 124: 53-68. [Crossref]

23. Levy J, Goldstein A, Zagoory-Sharon O, Schneiderman I, Eidelman-Rothman M, et al. (2016) Oxytocin selectively modulates brain response to stimuli probing social synchrony. Neuroimage 124: 923-930. [Crossref]

24. Verty AN, McFarlane JR, McGregor IS, Millet PE (2004) Evidence for an interaction between CB1 cannabinoid and oxytocin receptor in food and water intake. Neuropharmacology 47: 593-603.

25. Ross MG, Leake RD, Stegner H, Ervin G, Fisher DA (1985) Oxytocin release induced by melatonin in the ewe. Dev PharmacolTher 8: 254-259. [Crossref]

26. Lissoni P, Resentini M, Mauri R, Esposti D, Esposti G, et al. (1986) Effects of tetrahydrocannabinol on melatonin secretion in man. HormMetab Res 18: 77-78. [Crossref]

27. Evrard A, Hober C, Racadat A, Lefevre J, Wantyghem MC (1999) Atrial natriuretic hormone and and endocrine functions. Ann BiolClin (Paris) 57: 149-155. [Crossref]

28. Grant K, Loizidou M, Taylor I (2003) Endothelin-1: a multifunctional molecule in cancer. Br J Cancer 88: 163-166. [Crossref]

29. Haanwinckez MA, Elias LK, Pavaretto AL, Gutkowska J, McCann SM, et al. (1995) Oxytocin mediates atrial natriuretic peptide release and natriures is after volume expansion in the rat. Proc AmAcad Sci USA 92: 7902-7906. [Crossref]

30. Riegger GA, Liebau G, Kochsiek K (1982) Antidiuretic hormone in congestive heart failure. Am J Med 72: 49-52. [Crossref] 
Lissoni P (2020) A review on the cognitive functions of basal ganglia, amygdala, hippocampus, habenula, nucleus accumbens, cerebellum, and pineal gland

31. Rajmohan V, Mohandas E (2007) The limbic system. IndPsychiatry 49: 132-139. [Crossref]

32. Zald DH (2003) The human amygdala and the emotional evaluation of sensory stimuli. Brain Res Brain Res Rev 41: 88-123. [Crossref]

33. Ide S, Kakeda S, Korogi Y (2015) Anatomy of the thalamus. Brain Nerve 67: 14591469. [Crossref]

34. Rizzolatti G, Craighero L (2004) The mirror-neuron system. AnnuRevNeurosci 27 169-192. [Crossref]

35. Reiter RJ (2004) Mechanisms of cancer inhibition by melatonin. J Pineal Res 37: 213214. [Crossref]
36. Cassoni P, Marrocco T, Deaglio S, Sapino A, Bussolati G (2001) Biological relevance of oxytocin and oxytocin receptors in cancer cells and primary tumors. Ann Oncol 12: S37-S39. [Crossref]

37. Kong X, Wang X, Xu W, Behera S, Hellermann G, et al. (2008) Natriuretic peptide receptor A as a novel anticancer target. Cancer Res 68: 249-256. [Crossref]

38. Alonso G (2009) Vasopressin and angiogenesis. J SocBiol 203: 39-47. [Crossref]

39. Liang X, Liu R, Chen C, Ji F, Li T (2016) Opioid system modulates the immune function: a review. Transl Periop PainMed 1: 5-13. [Crossref]

40. Kosfeld M, Heinriches M, Zak PJ, Fischbacher U, Fehr E (2005) Oxytocin increases trust in humans. Nature 435: 673-676. [Crossref]

Copyright: $\bigcirc 2020$ Lissoni P. This is an open-access article distributed under the terms of the Creative Commons Attribution License, which permits unrestricted use, distribution, and reproduction in any medium, provided the original author and source are credited. 